\title{
Factors associated with folic acid supplementation in women presenting for antenatal care
}

\author{
S. Cawley ${ }^{1}$, L. Mullaney ${ }^{1}$, A. McKeating ${ }^{2}$, M. Farren ${ }^{2}$, D. McCartney ${ }^{1}$ and M.J. Turner ${ }^{2}$ \\ ${ }^{1}$ School of Biological Sciences, Dublin Institute of Technology, Dublin 8, Republic of Ireland and ${ }^{2} U C D$ Centre for \\ Human Reproduction, Coombe Women and Infants University Hospital, Dublin 8, Republic of Ireland
}

Neural tube defects (NTDs) are major congenital malformations that are potentially preventable if the woman takes periconceptional folic acid (FA) supplements. ${ }^{1}$ A recent report found that NTD incidence had increased in Ireland. ${ }^{2}$ The scientific evidence indicates that it takes an average of 12 weeks of supplementation with $400 \mu \mathrm{g}$ FA to achieve the desired red cell folate (RCF) level of 906 nmols. ${ }^{3,4}$ This is the level of RCF associated with a reduced risk of NTDs. ${ }^{5}$

This observational study examined the usage of FA supplementation in women presenting for antenatal care in a large, Dublin maternity hospital.

Women were recruited at their convenience in the first trimester. Their clinical and sociodemographic details were computerized. Maternal weight and height were measured before calculating body mass index. Detailed FA questionnaires were completed under the supervision of a trained researcher.

While $96 \cdot 1 \%(n=564)$ out of 587 reported that they took FA after they became pregnant, only $24 \cdot 7 \%(n=145)$ took it for 12 weeks preconceptionally as recommended. Only $5.7 \%(n=6)$ of obese women took high-dose FA as recommended. On univariate analysis, the strongest predictors of twelve weeks of preconceptional FA supplementation were higher maternal age, higher education and income, being married, being nulliparous, not smoking, infertility treatment and planned pregnancy. On multivariate analysis, both planned pregnancy and nulliparity were the most important predictors of preconceptional FA use.

When questioned in an open-ended manner; "Why do you take folic acid?" only $40.8 \%(n=230)$ of the women who took FA during the pregnancy mentioned the words "neural tube defect" or "spina bifida" or "anencephaly" as being the reason for taking FA. Those who were aware of the importance of FA for NTD prevention were more likely to be older, multiparous, to have planned their pregnancy, spent longer living in Ireland and spent longer in full time education (all $\mathrm{p}<0.05)$. When multivariate analysis was performed,the strongest predictors of having appropriate knowledge about the reasons for FA supplementation were parity and education.

Our study shows that recommendations to prevent NTDs by FA supplementation pre-pregnancy are not being properly implemented in Ireland. These findings also indicate that public health campaigns need to be strengthened to improve women's knowledge about FA's role in NTD prevention. It also shows that preconceptional FA supplementation practices are less favourable among low SES groups, and amongst women with an unplanned pregnancy. We recommend a review of current public health policies on FA supplementation, particularly in relation to vulnerable groups of women.

1. MRCVitaminStudyResearchGroup. (1991) Lancet 338 (8760):131-7

2. McDonnell R, Delaney V, O’Mahony MT, Mullaney C, Lee B, Turner MJ. (2014) Journal of Public Health 37(1):57-63.

3. Berti C, Biesalski HK, Gärtner R, Lapillonne AK. Pietrzik K, Poston L, Redman C, Koletzko I, Cetin A, (2011) Clin Nutr 30, 689-701.

4. Crider KS 1, Devine O, Hao L, Dowling DF, Li S 4, Molloy AM, Li Z, Zhu J, Berry RJ (2014) BMJ 349:g4554 doi: 10.1136/bmj.g4554

5. Daly LE, Kirke PN, Molloy A, Weir DG, Scott JM. (1995) JAMA 274, 1698-702. 